\title{
Displacements, Strains, and Stresses Investigations in an Inhomogeneous Rotating Hollow Cylinder Made of Functionally Graded Materials under an Axisymmetric Radial Loading
}

\author{
Elise Rose Atangana Nkene ${ }^{1,2}$, Luc Leroy Mambou Ngueyep ${ }^{3,4}$, Joseph Ndop, \\ Emma Sandring Djiokeng3, Jean-Marie Bienvenu Ndjaka ${ }^{3}$
}

${ }^{1}$ Department of Physics, Faculty of Sciences, University of Douala, Douala, Cameroon

${ }^{2}$ Douala Institute of Technology, Douala, Cameroon

${ }^{3}$ Laboratory of Material Sciences, Department of Physics, Faculty of Sciences, University of Yaoundé 1, Yaoundé, Cameroon

${ }^{4}$ Department of Mine, Mineral Processing and Environment, School of Geology and Mining Engineering, University of

Ngaoundéré, Meiganga, Cameroon

Email: *mamboulucleroy@gmail.com

How to cite this paper: Atangana Nkene, E.R., Mambou Ngueyep, L.L., Ndop, J., Djiokeng, E.S. and Ndjaka, J.-M.B. (2018) Displacements, Strains, and Stresses Investigations in an Inhomogeneous Rotating Hollow Cylinder Made of Functionally Graded Materials under an Axisymmetric Radial Loading. World Journal of Mechanics, 8, 59-72.

https://doi.org/10.4236/wjm.2018.83005

Received: February 2, 2018

Accepted: March 5, 2018

Published: March 9, 2018

Copyright (c) 2018 by authors and Scientific Research Publishing Inc. This work is licensed under the Creative Commons Attribution International License (CC BY 4.0).

http://creativecommons.org/licenses/by/4.0/

(c) $\underset{\mathrm{EY}}{\mathrm{i}}$ Open Access

\begin{abstract}
In this paper, an analytical and numerical study of strain fields, stress fields and displacements in a rotating hollow cylinder, whose walls were completely made in Functionally Graded Materials (FGM), was conducted. We have considered the rotating hollow cylinder submitted to an asymmetric radial loading. It is assumed that, because of the functional graduation of the material, the mechanical properties such as Young elastic modulus and the density varies in the radial direction, in accordance with a the power law function. The inhomogeneity parameter was selected between -1 and 1 . On the basis of the second law of Newton, Hooke's law and the strain-stress relationship, we established the differential equation which governs the equilibrium for a rotating hollow cylinder. We found the analytical solution and compared to the numerical solution obtained by using the shooting method and the fourth order Runge-Kutta algorithm. The analytical and numerical results lead to the conclusion that the magnitude of the tangential stresses is greater than that of the radial stresses. The changes due to the graduation of FGM does not produce consistent variations in the distribution of radial stresses, but strongly affects the distribution of tangential stresses. The tangential stresses, tangential strains and displacements are much higher at the inner surface of the cylinder wall. The internal radial pressure intensely affects the radial stresses and
\end{abstract}


the radial strain.

\section{Keywords}

Functionally Graded Materials, Radial Stresses, Tangential Stresses, Cylinder Wall

\section{Introduction}

Rotary devices such as disks, cylinders and even spheres have been widely used in mechanical applications and engineering including steam turbines and gas rotors, turbine generators and jet engines, internal combustion engines, boat propellers or even reciprocating and centrifugal compressors [1]-[15]. In such mechanical applications, the rotating hollow cylinder is often subject to different requirements with diverse characteristics. Some industrial demands require materials whose performance varies depending on space. It is difficult to find conventional materials that simultaneously meet such requirements. The development of "Functionally Graded Materials (FGM)" enabled to propose solutions to such problems.

Indeed FGM is a new class of materials that has really emerged in the second half of the 90's years. They are composite materials in which physical, chemical and mechanical properties vary continuously and which exhibit no discontinuity [1] [2] [7] [8] [9] [11]. The profile of variation of these properties depends on the area it is based and on a continual variation of their local composition as a function of space. We must remember that this form of material construction is observed even in nature. Thus the "Bamboo Tree" presents a variation in its natural density in fiber, which gives it the ability to optimally support charges when it undergoes the pressure of winds [3] [4]. Similarly when viewed in the cross section of the tree trunk, we note that its density varies from young wood (core) to the old wood (outer part). This density variation in the wood is simply due to the natural transition of the small pores in the old wood to larger pores in the young wood [5]. It is therefore believed that the hollow cylinders, built from highly suitable FGM can provide solutions to the demands of the diverse characteristics or requirements to which they are subjected in their various applications. The optimization of the modeling and design of homogeneous hollow cylinder, for example to reduce the risk of accidents and disfunctioning of systems in which they are been integrated, require the understanding of their elasto-plastic regime. This topic has been so far extensively treated. Different analytical and numerical investigations were carried out in this context [1]-[14]. However the estimation of strain and stress fields and displacement in the rotating hollow cylinders constructed on the basis of the FGM is an important and actual topic in engineering, but remains poorly investigated.

The main objective of this work is to find analytical and numerical solutions for the case of a rotating hollow cylinder whose walls are entirely made on Func- 
tionally Graded Materials and submitted to internal and external radial loading.

Analytical and numerical solutions obtained show clearly the effects of different profiles describing the graduation of the material properties. This is the case for the strain fields, stress fields and displacement.

The paper is organized as follows. In the first part we present the model studied. In the second part the equations describing the model are calculated and resolved. The third part presents the analytical and numerical results before concluding the work in the fourth part.

\section{Method}

\subsection{Problem Formulation}

In this part, we analyze the stress and strain states of a rotating hollow cylinder submitted to non-axisymmetric pressure. Let us consider a long hollow FGM circular cylinder with inner radius $a$ and outer radius $b$ subjected to the action of a uniform internal and external pressure loading respectively $P_{i}$ and $P_{0}$, as show in Figure 1.

The most common functionally graded materials in such applications are made of ceramic/metal non homogeneous structure, in which ceramic provides good thermal resistance and metal roles as a superior toughness and hardness [7] [8] [9] [10] [11]. Therefore, as it was experimentally demonstrated [6], a FGM is nonhomogeneous in composition and so its properties, especially those of Young modulus of elasticity, thermal conductivity, and mass density may vary continuously through the material. Since the modulus of elasticity, $E$, is important in the determination of the strength of the structural element during operation, it is of engineering interest to ascertain the effect of variable modulus of elasticity on the deformation behavior of basic structures [9]. Poisson's ratio is assumed to be constant because his variation has less practical significance than that of the elastic modulus and density [16]. In this work the materials are assumed to be linearly elastic and isotropic and the modulus of elasticity of the cylindrical material depends only on the radial direction. We assume as Horgan that the Young modulus of elasticity and the density are given by a power law function [17]

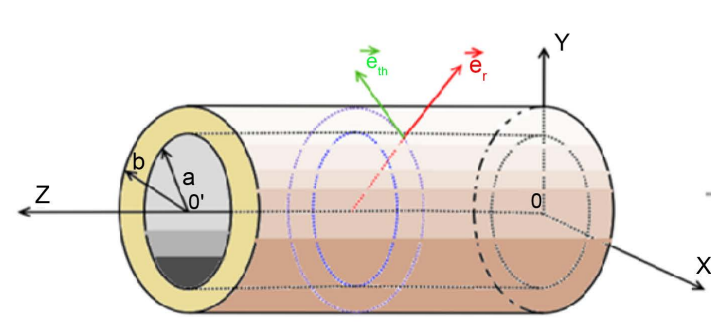

(a)

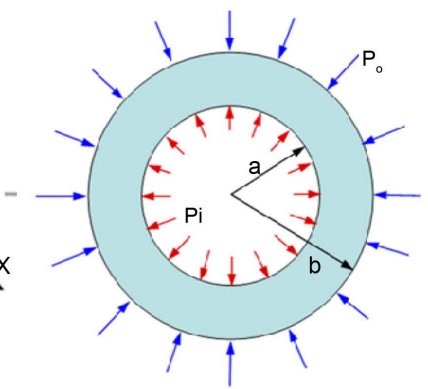

(b)

Figure 1. (a) Hollow FGM circular cylinder; (b) thick circular cylinder under non-axisymmetric loading. 


$$
E(r)=E_{0}\left(\frac{r}{b}\right)^{n} ; \rho(r)=\rho_{0}\left(\frac{r}{b}\right)^{n}
$$

where $E_{0}$ is the reference value of $E, r$ is the radial coordinate and the exponent $n$ is a positive or negative real number, reflecting the degree of non-uniformity of the material.

Due to the axisymmetric load condition of hollow cylinder, the circumferential component of displacement is zero, and the radial component $u$ depends on the radial distance $r$ only [17]. Correspondingly, the stress components $\sigma_{r}$ and $\sigma_{\theta}$ are also $r$-dependent only. In this work we are interested by the plane strain and we suppose that the length of hollow cylinder is very high compared to the radius in order to neglect the boundary effects.

\subsection{Stress and Strain Analysis}

For the rotating hollow cylinder, the equation of equilibrium is [13]-[18]:

$$
\frac{\mathrm{d}}{\mathrm{d} r}\left(r \sigma_{r}\right)-\sigma_{\theta}+\rho(r) \omega^{2} r^{2}=0
$$

where $\sigma_{r}$ and $\sigma_{\theta}$ are the radial and circumferential components of stress, $\omega$ is the angular velocity of the cylinder, $\rho$ the density of the material is assumed to be constant.The strain-stress's relation for infinitesimally elastic deformation (Hooke's law) is given by:

$$
\overline{\bar{\sigma}}=\lambda \operatorname{Tr}(\overline{\bar{\varepsilon}}) \overline{\bar{I}}+2 \mu \overline{\bar{\varepsilon}}
$$

where the Lame's constant is given by:

$$
\lambda=\frac{E v}{(1+v)(1-2 v)} ; \mu=\frac{E}{2(1+v)}
$$

In the case of small deformations and due to the rotational symmetry, the strain-displacement relations are given by:

$$
\overline{\bar{\varepsilon}}=\left(\begin{array}{ccc}
\varepsilon_{r}=\frac{\mathrm{d} u_{r}}{\mathrm{~d} r} & 0 & 0 \\
0 & \varepsilon_{\theta}=\frac{u_{r}}{r} & 0 \\
0 & 0 & \varepsilon_{z}
\end{array}\right)
$$

By using Equations (1), (3), (4) and (5), the stress-strain relation can become:

$$
\left\{\begin{array}{l}
\sigma_{r}=\frac{E_{0}}{(1+v)(1-2 v)}\left(\frac{r}{b}\right)^{n}\left[v \varepsilon_{z}+\frac{v u_{r}}{r}+(1-v) \frac{\mathrm{d} u_{r}}{\mathrm{~d} r}\right] \\
\sigma_{\theta}=\frac{E_{0}}{(1+v)(1-2 v)}\left(\frac{r}{b}\right)^{n}\left[v \varepsilon_{z}+\frac{(1-v) u_{r}}{r}+v \frac{\mathrm{d} u_{r}}{\mathrm{~d} r}\right] \\
\sigma_{z}=E_{0}\left(\frac{r}{b}\right)^{n} \varepsilon_{z}+v\left(\sigma_{r}+\sigma_{\theta}\right)
\end{array}\right.
$$

Substituting Equation (6) into Equation (2), the equation of displacement is 
given by:

$$
r \frac{\mathrm{d}^{2} u_{r}}{\mathrm{~d} r^{2}}+(n+1) \frac{\mathrm{d} u_{r}}{\mathrm{~d} r}+\frac{[v(n+1)-1]}{r(1-v)} u_{r}+\frac{n v \varepsilon_{z}}{1-v}+\frac{(1+v)(1-2 v) \rho_{0} \omega^{2}}{E_{0}(1-v)} r^{2}=0
$$

This equation can be written as:

$$
r^{2} \frac{\mathrm{d}^{2} u_{r}}{\mathrm{~d} r^{2}}+(n+1) r \frac{\mathrm{d} u_{r}}{\mathrm{~d} r}-\frac{[1-v(n+1)]}{(1-v)} u_{r}=-\frac{n r v \varepsilon_{z}}{1-v}-\frac{(1+v)(1-2 v) \rho_{0} \omega^{2}}{E_{0}(1-v)} r^{3}
$$

We can note that for a homogenous material $(n=0)$, we obtain the governing differential equation found in the literature for a hollow rotating cylinder [4]-[10].

$$
\left\{\begin{array}{l}
r^{2} \frac{\mathrm{d}^{2} u_{r}}{\mathrm{~d} r^{2}}+r \frac{\mathrm{d} u_{r}}{\mathrm{~d} r}-u_{r}=-\frac{(1+v)(1-2 v) \rho_{0} \omega^{2}}{E_{0}(1-v)} r^{3} ; n=0 ; \omega \neq 0 \\
r^{2} \frac{\mathrm{d}^{2} u_{r}}{\mathrm{~d} r^{2}}+r \frac{\mathrm{d} u_{r}}{\mathrm{~d} r}-u_{r}=0 ; n=0 ; \omega=0
\end{array}\right.
$$

The next part of this work is devoted to the analytical and numerical resolution of Equation (8)

\subsection{Analytical and Numerical Solution}

\section{Analytical Solution}

To find exacts solutions to displacements, strain and stress, we rewrite the Equation (8) in a series of three differential equations. One of these equations is homogenous and the others are inhomogeneous.

The Equation (8) becomes:

$$
\left\{\begin{array}{l}
r^{2} \frac{\mathrm{d}^{2} u_{r}}{\mathrm{~d} r^{2}}+(n+1) r \frac{\mathrm{d} u_{r}}{\mathrm{~d} r}-\frac{[1-v(n+1)]}{(1-v)} u_{r}=0 \\
r^{2} \frac{\mathrm{d}^{2} u_{r}}{\mathrm{~d} r^{2}}+(n+1) r \frac{\mathrm{d} u_{r}}{\mathrm{~d} r}-\frac{[1-v(n+1)]}{(1-v)} u_{r}=-\frac{n r v \varepsilon_{z}}{1-v} \\
r^{2} \frac{\mathrm{d}^{2} u_{r}}{\mathrm{~d} r^{2}}+(n+1) r \frac{\mathrm{d} u_{r}}{\mathrm{~d} r}-\frac{[1-v(n+1)]}{(1-v)} u_{r}=-\frac{(1+v)(1-2 v) \rho_{0} \omega^{2}}{E_{0}(1-v)} r^{3}
\end{array}\right.
$$

1) Resolution of homogenous equation

The homogenous equation is given by:

$$
r^{2} \frac{\mathrm{d}^{2} u_{r}}{\mathrm{~d} r^{2}}+(n+1) r \frac{\mathrm{d} u_{r}}{\mathrm{~d} r}-\frac{[1-v(n+1)]}{(1-v)} u_{r}=0
$$

To solve the Equation (11), we make the following change of variable:

$$
r=\mathrm{e}^{\lambda}
$$

By using Equation (12), Equation (11) becomes:

$$
\frac{\mathrm{d}^{2} u}{\mathrm{~d} \lambda^{2}}+n \frac{\mathrm{d} u}{\mathrm{~d} \lambda}-\frac{[1-v(n+1)]}{1-v} u=0
$$

The solution of this equation is given by: 


$$
u_{H}=C_{1} r^{-(n+K) / 2}+C_{2} r^{-(n-K) / 2}, C_{1}, C_{2} \in \mathfrak{R}
$$

where $K=\sqrt{n^{2}+4-\frac{4 n v}{1-v}}$ and the integration constants are $C_{1}$ and $C_{2}$

\section{2) Resolution of inhomogeneous equations}

According to Equation (10) the first inhomogeneous equation is given by:

$$
r^{2} \frac{\mathrm{d}^{2} u_{r}}{\mathrm{~d} r^{2}}+(n+1) r \frac{\mathrm{d} u_{r}}{\mathrm{~d} r}-\frac{[1-v(n+1)]}{(1-v)} u_{r}=-\frac{n v \varepsilon_{z}}{1-v} r
$$

To solve this equation, we search the particular solution $u_{P 1}$ which has the same form as the second member of the equation:

$$
u_{P 1}=A r+B
$$

where $A$ and $B$ are the constants.

By substituting Equation (16) into Equation (15) we obtain:

$$
A=-v \varepsilon_{z} ; B=0
$$

Then Equation (16) becomes:

$$
u_{P 1}=-v \varepsilon_{z} r
$$

According to Equation (10) the second inhomogeneous equation is given by:

$$
r^{2} \frac{\mathrm{d}^{2} u_{r}}{\mathrm{~d} r^{2}}+(n+1) r \frac{\mathrm{d} u_{r}}{\mathrm{~d} r}-\frac{[1-v(n+1)]}{(1-v)} u_{r}=-\frac{(1+v)(1-2 v) \rho_{0} \omega^{2}}{E_{0}(1-v)} r^{3}
$$

To solve Equation (19) we search the particular solution $u_{P 2}$ which has the same form as the second member of the equation:

$$
u_{P 2}=D r^{3}
$$

where $D$ is constant.

By substituting Equation (20) into Equation (19) we obtain the constant:

$$
D=-\frac{(1+v)(1-2 v) \rho_{0} \omega^{2}}{[8+3 n-2(4+n) v] E_{0}}
$$

By substituting Equation (21) into Equation (20) we obtain:

$$
u_{P 2}=-\frac{(1+v)(1-2 v) \rho_{0} \omega^{2}}{[8+3 n-2(4+n) v] E_{0}} r^{3}
$$

The general solution of Equation (10) is given by:

$$
u_{r}=C_{1} r^{-(n+K) / 2}+C_{2} r^{-(n-K) / 2}-\frac{(1+v)(1-2 v) \rho_{0} \omega^{2}}{[8+3 n-2(4+n) v] E_{0}} r^{3}-v \varepsilon_{z} r
$$

In the case of homogenous material, Equation (23) becomes:

$$
\left\{\begin{array}{l}
u_{r}=C_{1} r^{-1}+C_{2} r-v \varepsilon_{z} r ; n=0 ; \omega=0 \\
u_{r}=C_{1} r^{-1}+C_{2} r-\frac{(1+v)(1-2 v) \rho_{0} \omega^{2}}{8(1-v) E_{0}} r^{3}-v \varepsilon_{z} r ; n=0 ; \omega \neq 0
\end{array}\right.
$$


By substituting Equation (24) into Equation (5) the components of strain tensor are given by:

$$
\left\{\begin{array}{l}
\varepsilon_{r}=-C_{1} \frac{(n+K)}{2} r^{-(n+K+2) / 2}-C_{2} \frac{(n-K)}{2} r^{-(n-K+2) / 2}-\frac{3(1+v)(1-2 v) \rho_{0} \omega^{2}}{[8+3 n-2(4+n) v] E_{0}} r^{2}-v \varepsilon_{z} \\
\varepsilon_{\theta}=C_{1} r^{-(n+K+2) / 2}+C_{2} r^{-(n-K+2) / 2}-\frac{(1+v)(1-2 v) \rho_{0} \omega^{2}}{[8+3 n-2(4+n) v] E_{0}} r^{2}-v \varepsilon_{z}
\end{array}\right.
$$

By substituting Equation (22) into Equation (6), the components of stress tensor are given by:

$$
\left\{\begin{array}{l}
\sigma_{r}=-\frac{E_{0}}{2(1+v)(1-2 v) b^{n}} r^{\frac{(n-K-2)}{2}}\left\{C_{1}[(K+n)(1-v)-2 v]+C_{2} r^{K}[(n-K)(1-v)-2 v]\right\}-\frac{(3-2 v) \rho_{0} \omega^{2}}{8+3 n-2 v(4+n) b^{n}} r^{n+2} \\
\sigma_{\theta}=-\frac{E_{0}}{2(1+v)(1-2 v) b^{n}} r^{\frac{(n-K-2)}{2}}\left\{C_{1}[-2+(K+n+2) v]+C_{2} r^{K}[-2+(n-K+2) v]\right\}-\frac{[1+2 v] \rho_{0} \omega^{2}}{8+3 n-2 v(4+n) b^{n}} r^{n+2} \\
\sigma_{z}=-\frac{v E_{0}}{2(1+v)(1-2 v) b^{n}} r^{\frac{(n-K-2)}{2}}\left\{C_{1}(K+n-2)+C_{2}(K-n-2) r^{K}\right\}-\frac{4 v \rho_{0} \omega^{2}}{8+3 n-2 v(4+n) b^{n}} r^{n+2}+\frac{r^{n} \varepsilon_{z} E_{0}}{b^{n}}
\end{array}\right.
$$

These solutions don't exist when $n=n_{c}=-\frac{8(1-v)}{3-2 v}$.

\section{3) Boundary conditions}

The boundary conditions are used to determine the constants $C_{1}$ and $C_{2}$. For the hollow cylinder submitted to uniform pressures $P_{0}$ and $P_{i}$ on the inner and outer surfaces respectively, the mechanical boundary conditions can be expressed as:

$$
\sigma_{r}(b)=-P_{0} ; \sigma_{r}(a)=-P_{i}
$$

By substituting Equation (27) into Equation (26), the constants are given by:

$$
\left\{\begin{array}{l}
C_{1}=-\frac{2(1+v)(1-2 v)}{[8+3 n-2 v(4+n)][(n+K)(1-v)-2 v] E_{0}\left(a^{K}-b^{K}\right)}\left\{C_{11}+C_{12}+C_{13}\right\} \\
C_{2}=-\frac{2(1+v)(1-2 v)}{[8+3 n-2 v(4+n)][(n-K)(1-v)-2 v] E_{0}\left(a^{K}-b^{K}\right)}\left\{C_{21}+C_{22}+C_{23}\right\}
\end{array}\right.
$$

where

$$
\left\{\begin{array}{l}
C_{11}=-[8+3 n-2 v(4+n)] P_{i} a^{(2+K-n) / 2} b^{(n+K)} \\
C_{12}=[8+3 n-2 v(4+n)] P_{0} a^{K} b^{(n+K+2) / 2} \\
C_{13}=\left\{(3-2 v)\left(b^{(n+K)} a^{(n+K+6) / 2}-a^{K} b^{(3 n+K+6) / 2}\right)\right\} \rho_{0} \omega^{2} \\
C_{21}=[8+3 n-2 v(4+n)] P_{o} b^{(2+K+n) / 2} \\
C_{22}=-[8+3 n-2 v(4+n)] P_{i} b^{n} a^{(2+K-n) / 2} \\
C_{23}=\left\{(3-2 v)\left(a^{(n+K+6) / 2} b^{n}-b^{(3 n+K+6) / 2}\right)\right\} \rho_{0} \omega^{2}
\end{array}\right.
$$


In the case of homogenous hollow cylinder, Equation (29) becomes:

$$
\left\{\begin{array}{l}
C_{1}=\frac{\left[8(1-v)\left(P_{1}-P_{2}\right)+\left(b^{2}-a^{2}\right)(3-2 v) \rho \omega^{2}\right](1+v) a^{2} b^{2}}{8\left(a^{2}-b^{2}\right)(v-1) E_{0}} \\
C_{2}=-\frac{\left[8(1-v)\left(P_{1}-P_{2}\right)+\left(b^{2}-a^{2}\right)(3-2 v) \rho \omega^{2}\right](1+v)(2 v-1)}{8\left(a^{2}-b^{2}\right)(v-1) E_{0}} ; n=0, \omega \neq 0
\end{array} ;\right.
$$

In order to determine the constant $\varepsilon_{z}$, we use the expression of the z-component of the force given by:

$$
\begin{aligned}
F_{z}^{(\alpha, \beta)}= & \int_{\alpha}^{\beta} r \sigma_{z} \mathrm{~d} r=B_{1}\left[\alpha^{(n-K+2) / 2}-\beta^{(n-K+2) / 2}\right]+B_{2}\left[\alpha^{(n+K+2)}-\beta^{(n+K+2)}\right] \\
& +B_{3}\left[\alpha^{(n+4)}-\beta^{(n+4)}\right]+B_{4}\left[\alpha^{(n+2)}-\beta^{(n+2)}\right] \varepsilon_{z}
\end{aligned}
$$

where:

$$
\begin{aligned}
& B_{1}=-\frac{v E_{0}(K+n-2) C_{1}}{2(1+v)(1-2 v) b^{n}} ; B_{2}=-\frac{v E_{0}(K-n-2) C_{2}}{2(1+v)(1-2 v) b^{n}} ; \\
& B_{3}=-\frac{4 v \rho_{0} \omega^{2}}{8+3 n-2 v(4+n) b^{n}} ; B_{4}=\frac{E_{o}}{b^{n}}
\end{aligned}
$$

We suppose that the ends of the cylinders are free. It contracts as is rotates. The net force $F_{z}$ in the axial direction should be equal to be zero [8] [9] [10]. By solving Equation (31) we obtain:

$$
\begin{aligned}
\varepsilon_{z}= & -\frac{2(n+2) B_{1}}{(n-K+2) B_{4}}\left[\alpha^{-(n+K+2) / 2}-\beta^{-(n+K+2) / 2}\right] \\
& -\frac{2(n+2) B_{2}}{(n+K+2) B_{4}}\left[\alpha^{-(n-K+2) / 2}-\beta^{-(n-K+2) / 2}\right] \\
& -\frac{(n+2) B_{3}}{(n+4) B_{4}}\left[\alpha^{2}-\beta^{2}\right]
\end{aligned}
$$

\section{Numerical and Analytical Results}

In this part we study the influence of the inhomogeneous parameter $n$ on the stress, strain and displacement in the rotation hollow cylinder. We use the constants given by the properties of an aluminum alloy (7075-T6) [14]:

$$
\begin{aligned}
& a=40 \mathrm{~cm} ; b=60 \mathrm{~cm} ; v=0.3 ; E_{0}=79.0 \times 10^{9} \mathrm{~Pa} ; \\
& P_{o}=10^{5} \mathrm{~Pa} ; \rho_{0}=2800 \mathrm{~kg} \cdot \mathrm{m}^{-3} ; \omega=250 \mathrm{rad} \cdot \mathrm{s}^{-1} ; a \leq r \leq b
\end{aligned}
$$

To validate the analytical results we make the comparison with the numerical one. Equation (2) can be written as:

$$
\frac{\mathrm{d} \sigma_{r}}{\mathrm{~d} r}+\frac{\sigma_{r}-\sigma_{\theta}}{r}+\rho(r) \omega^{2} r=0
$$

The solution of this equation can be efficiently handled by using a special stress-function that automatically satisfies the equilibrium Equation (33). A par- 
ticular stress-stress function relation is given by:

$$
\sigma_{r}=\frac{F}{r} ; \sigma_{\theta}=\frac{\mathrm{d} F}{\mathrm{~d} r}+\rho(r) \omega^{2} r^{2},
$$

where $F=F(r)$ is the stress function. The Strain compatibility equation is given by:

$$
\varepsilon_{r}=\frac{\mathrm{d}}{\mathrm{d} r}\left(r \varepsilon_{\theta}\right)
$$

The strain-stress relation can be written:

$$
\left\{\begin{array}{l}
\varepsilon_{\theta}=\frac{1}{E(r)}\left(\sigma_{\theta}-v \sigma_{r}\right) \\
\varepsilon_{r}=\frac{1}{E(r)}\left(\sigma_{r}-v \sigma_{\theta}\right)
\end{array}\right.
$$

Substitution of Equations (34) and (36) in the compatibility relation (35) gives the following governing equation:

$$
r^{2} \frac{\mathrm{d}^{2} F}{\mathrm{~d} r^{2}}+r(1-n) \frac{\mathrm{d} F}{\mathrm{~d} r}-(1-v n) F=\frac{\rho \omega^{2} r^{n+3}(3+v)}{b^{n}} .
$$

with boundary conditions:

$$
F(a)=a P_{i} ; F(b)=b P_{o},
$$

the Equation (36) can be transformed as:

$$
Y_{2}^{\prime}=\frac{1-n}{r} Y_{2}+\frac{1-v n}{r^{2}} Y_{1}+\frac{\rho \omega^{2} r^{n+1}(3+v)}{b^{n}},
$$

where $Y_{1}=F ; Y_{2}=\frac{\mathrm{d} F}{\mathrm{~d} r}=F^{\prime}=f\left(r, Y_{1}, Y_{2}\right)$.

The Equation (36) is transformed into a set of coupled time-dependent discrete differential equations that are solved by using the shooting method and the fourth order Runge-Kutta algorithm. The analytical and numerical solutions are thus presented.

Figure 2 presents the variation of displacement along the radial direction of an inhomogeneous hollow cylinder.

In Figure 2, displacements decrease linearly along the radial direction. All displacements are higher at the inner surface, and vary with parallel curves in the radial direction of cylinder. In addition, the radial displacement increases when the inhomogeneous parameter $n$ increases. For a homogenous material, the maximum value of radial displacement is respectively $1.77 \times 10^{-4} \mathrm{~m}$ and 3.22 $\times 10^{-4} \mathrm{~m}$ for $P_{i}=10^{5} \mathrm{~Pa}$. We conclude that radial displacement decrease when the internal pressure increases. More ever the radial displacement curves save the parallelism when internal pressure $\mathrm{P}_{\mathrm{i}}$ and the inhomogeneous parameter $n$ changes. In Figure 2(b) the displacements are higher than in Figure 2(a), and then these displacements are much affected by the outer pressure than the internal one. 


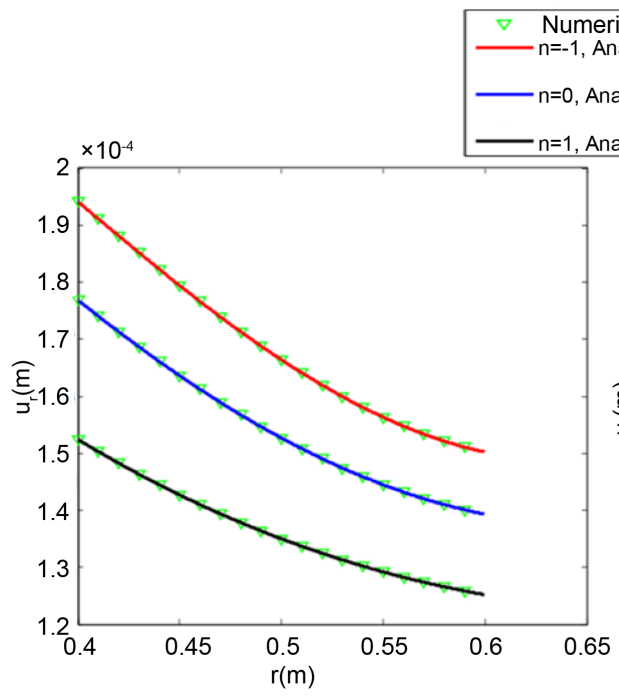

(a)

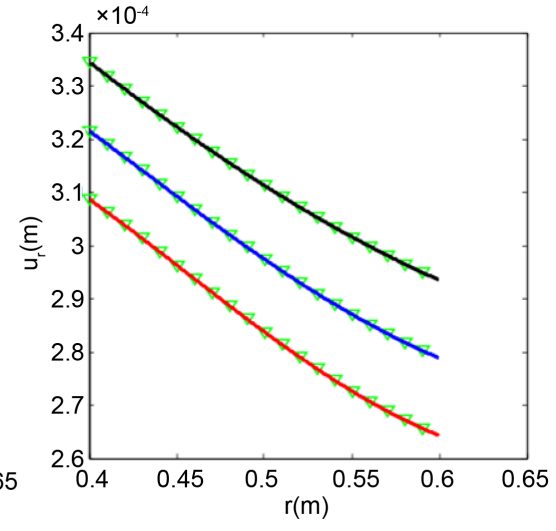

(b)

Figure 2. Displacement as function of radius $r$, (a) for $P_{i}=100 P_{o^{\prime}}$ (b) for $P_{i}=P_{o}$.

The variation of radial strain is depicted in Figure 3.

In Figure 3(a) we observe that radial strain increases gradually from the inner surface through the outer surface and decrease with the inhomogeneous parameter. However in Figure 3(b), all radial strains are higher at the outer surface, and vary with parallel curves in the radial direction of the cylinder. For $n=-1$ and $n=0$, we observe that the radial strain increases and then decreases along the radius.

For $n=1$, the radial strain decreases gradually along the radial direction and the maximum value of strain is $7.6 \times 10^{-4} \mathrm{~m}$.

Figure 4 illustrates the variation of tangential strain along the radial direction of inhomogeneous hollow cylinder.

In Figure 4, all tangential strains are higher at the inner surface, and vary with parallel curves in the radial direction of the cylinder. We observe that tangential strain decreases gradually from the inner surface through the outer surface.

For $\mathrm{Pi}=$ Po tangential strains increase with increasing inhomogeneous parameter and reaches to maximum value of $4.8 \times 10^{-4} \mathrm{~m}(n=-1)$. For $P_{\mathrm{i}}=100 \mathrm{Po}$, tangential strains decrease with increasing inhomogeneous parameter and the maximum value of tangential strain is $8.3 \times 10^{-4} \mathrm{~m}(n=1)$.

Figure 5 shows the variations of radial stresses $\sigma_{r}$ along the radial direction of inhomogeneous hollow cylinder, obtained from analytical and numerical solutions.

In Figure 5(a), the radial stress decreases gradually from the inner surface through the outer surface. The maximum value is $10 \mathrm{Mpa}$. From $r=0.4 \mathrm{~m}$ to $r=$ $0.52 \mathrm{~m}$, radial stress decrease when inhomogeneous parameter $n$ increases. From $r=0.52 \mathrm{~m}$, inhomogeneous parameter doesn't affect the radial stress. In Figure 5 (b), according to boundary conditions, radial stress I zero at the inner and outer surfaces. From $r=0.4 \mathrm{~m}$ to $r=0.5 \mathrm{~m}$, radial stress gradually increases and 


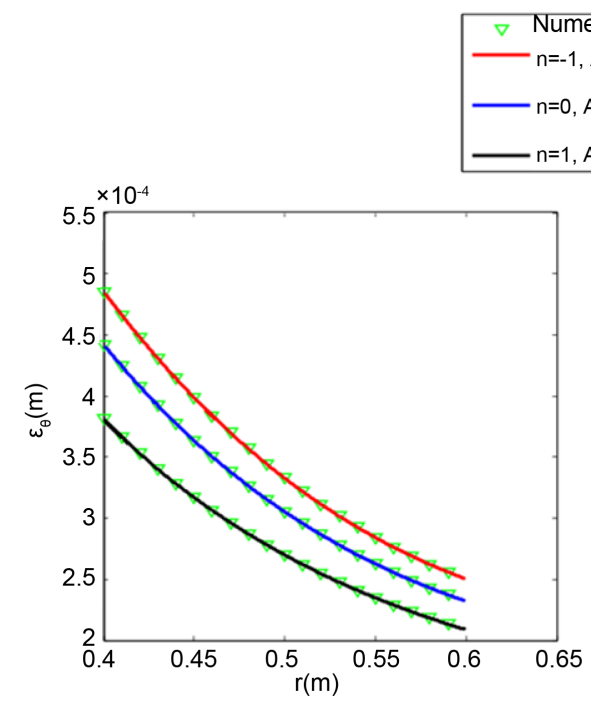

(a) umerical results

$=1$, Analytical result

$=0$, Analytical result

$=1$, Analytical result

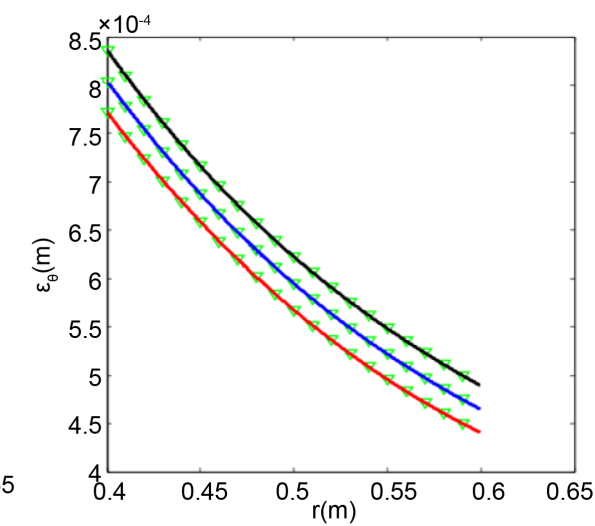

(b)

Figure 3. Radial strain as function of radius $r$ : (a) for $P_{i}=100 P_{o}$, (b) for $P_{i}=P_{o}$.

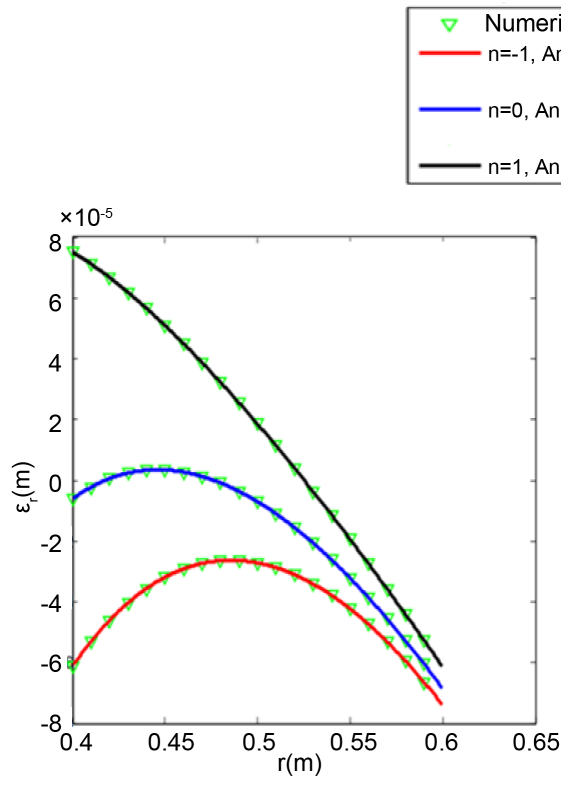

(a)

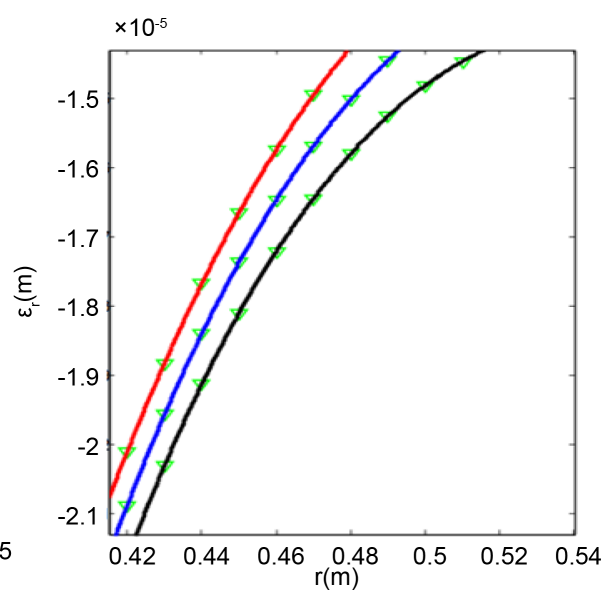

(b)

Figure 4. Tangential strain as function of radius $r$. (a) for $P_{i}=100 P_{o}$, (b) for $P_{i}=P_{o}$.

reaches to its maximum value $3.75 \mathrm{Mpa}$ at $r=0.5$ and then decrease to zero. In addition, radial stress decreases when inhomogeneous parameter $n$. They have lower value for $n=1$ when compared with the results for $n=0$ and $n=-1$. It is due to the fact that Young modulus of elasticity decreases when inhomogeneous parameter $(\mathrm{n})$ increases.

The variation of tangential stress obtained from analytical and numerical solutions is depicted in Figure 6.

In Figure 6, we observe that tangential stress decreases gradually from the inner surface through the outer surface. All tangential stresses are higher at the 


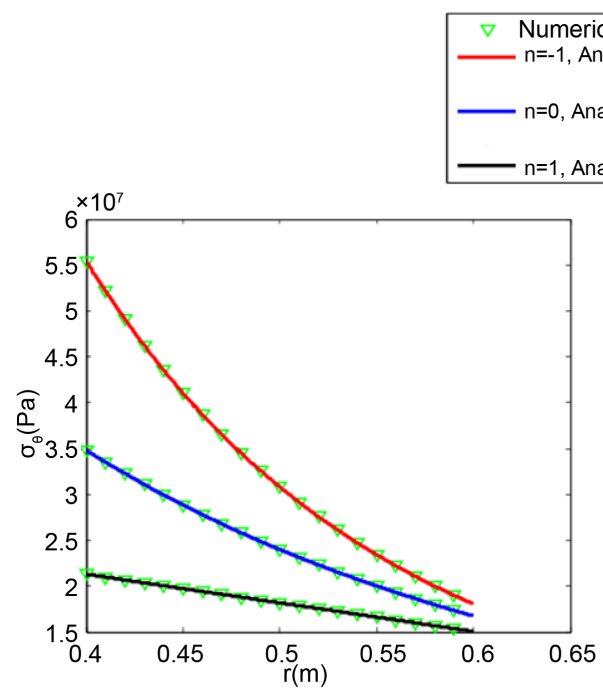

(a)

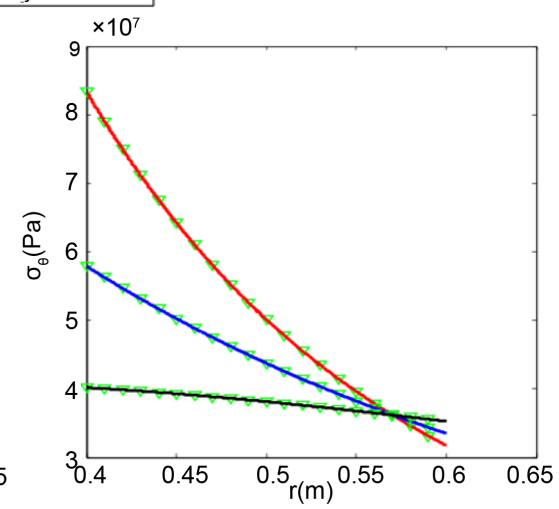

(b)

Figure 5. Radial stress as function of radius $r$. (a) for $P_{i}=100 P_{o}$, (b) for $P_{i}=P_{o}$.

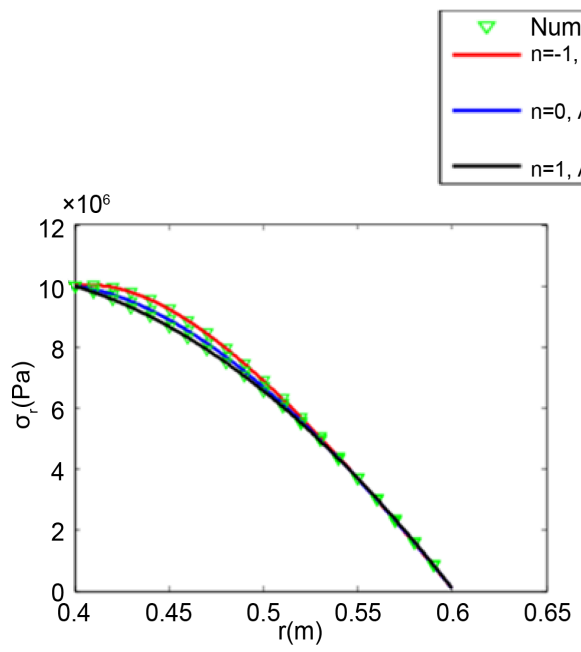

(a)

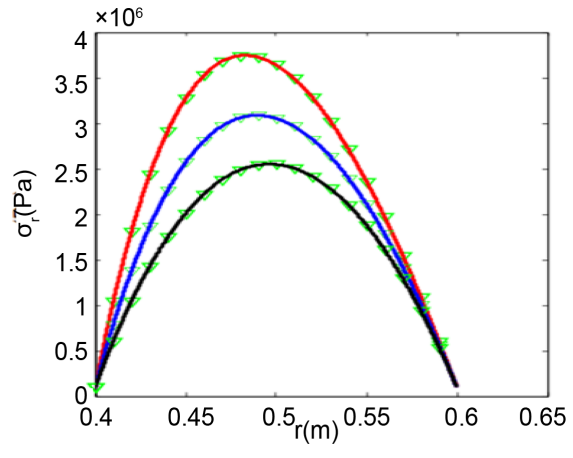

(b)

Figure 6. Tangential stress as function of radius $r$. (a) for $P_{i}=100 P_{o}$, (b) for $P_{i}=P_{o}$.

inner surface. For $P_{\mathrm{i}}=P_{o}$, tangential stress decreases with increasing inhomogeneous parameter and reaches to its maximum value of $55 \mathrm{Mpa}(\mathrm{n}=-1)$. For $P_{\mathrm{i}}=$ $100 P_{\mathrm{o}}$, tangential strains decrease with increasing inhomogeneous parameter and maximum value of tangential stress is $82 \mathrm{Mpa}(n=-1)$. They have lower value for $n=1$ when compared with the results for $n=0$ and $n=-1$.

\section{Conclusions}

In this paper we analyze stress and strains along the radial direction of inhomogeneous hollow rotating cylinder under axisymmetric radial loading. Analytical and numerical results lead us to conclude that:

- The stress, strain and displacement obtained from analytical and numerical solution are in good correlation. 
- The magnitudes of tangential stress are higher than those of radial stress.

- Change in the gradient of the FGM tube does not produce a substantial variation of the radial stress, but strongly affects the distribution of the tangential stress.

- Tangential stress, tangential strain and displacements are higher at the inner surface.

- Internal radial pressure strongly affects the radial stresses and radial strain.

The results obtained are helpful in designing FGM cylindrical vessels to prevent failure. This investigation permits us to optimize the elastic response of cylinders under pressure by tailoring the thickness variation of the elastic properties and to reduce manufacturing costs given by the technological limitations that occur to produce entire functionally graded walls.

\section{References}

[1] Ndop, J. (2000) Mechanische Charakterisierung gradierter Materialien mit der Raster Ultraschallmikroskopie mit Vektorkontrast, Dissertation Fakultaet fuer Physik und Geowissenschaften, Universitaet Leipzig, Germany.

[2] Suresh, S. and Mortensen, A. (1998) Fundamentals of Functionally Graded Materials, Book 698, IOM Communications Ltd.

[3] Amada, S., Munekata, T., Nagase, Y. and Shimizu, N. (1995) Hierarchical Gradient Structure of Bamboo. In: Ilschner, B. and Cheraldi, N., Eds., Pro. 3rd International Symposium on Functionally Graded Materials, Presses Polytechniques Etuniversitaires Romandes, Lausanes.

[4] Nogata, F., Matsui, K., Kagechika, K., Sueyoshi, Y. and Tomita, K. (1999) Estimation of In Vivo Bone Mineral Density (BMD) and Shape Characterization for Diagnosing Osteoporosis by Ultrasonic Inspection. Journal of Biomechanical Engineering, 121, 298-303.

[5] Parviz, N. (1995) Wood Multilevel Gradient Structure. In: Ilschner, B. and Cheraldi, N., Eds., Pro. 3 rd International Symposium on Functionally Graded Materials, Presses Polytechniques et Universitaires Romandes, Lausanes.

[6] Ndop, J., Kim, T.J. and Grill, W. (1999) Mechanical Characterization of Graded Materials by Ultrasonic Microscopy with Phase Contrast. Materials Science Forum, 308-311, 873878. https://doi.org/10.4028/www.scientific.net/MSF.308-311.873

[7] Mahamood, R.M., Akinlabi, E.T., Shukla, M. and Pityana, S. (2012) Functionally Graded Material: An Overview. Proceedings of the World Congress on Engineering 2012, Vol. III, London, 4-6 July 2012.

[8] Makwana, A.B. and Panchal, K.C. (2014) A Review of Stress Analysis of Functionally Graded Material Plate with Cut-out. International Journal of Engineering Research and Technology (IJERT), 3.

[9] Sburlati, R. (2012) Analytical Elastic Solutions for Pressurized Hollow Cylinders with Internal Functionally Graded Coatings. Composite Structures, 94, 3592-3600. https://doi.org/10.1016/j.compstruct.2012.05.018

[10] Tutanku, N. (2007) Stresses in Thick-Walled FGM Cylinders with ExponentiallyVarying Properties. Engineering Structures, 29, 2032-2035. https://doi.org/10.1016/j.engstruct.2006.12.003

[11] Peng, X.L. and Li, X.F. (2010) Thermal Stress in Rotating Functionally Graded Hol- 
low Circular Disks. Composite Structures, 92, 1896-1904.

https://doi.org/10.1016/j.compstruct.2010.01.008

[12] Jahromi, B.H., Nayeb-Hashemi, H. and Vaziri, A. (2012) Elasto-Plastic Stresses in a Functionally Graded Rotating Disk. Journal of Engineering Materials and Technology.

[13] Eraslan, A.N. (2003) Elastic-Plastic Deformations of Rotating Variable Thickness Annular Disks Withfree, Pressurized and Radially Constrained Boundary Conditions. International Journal of Mechanical Sciences, 45, 643667. https://doi.org/10.1016/S0020-7403(03)00112-7

[14] Çallioğlu, H., Bektaş, N.B. and Sayer, M. (2011) Stress Analysis of Functionally Graded Rotating Discs: Analytical and Numerical Solutions. Acta Mechanica Sinica, 27, 950-955. https://doi.org/10.1007/s10409-011-0499-8

[15] Reddy, J.N. (2000) Analysis of Functionally Graded Materials. International Journal for Numerical Methods in Engineering, 47, 663-684.

https://doi.org/10.1002/(SICI)1097-0207(20000110/30)47:1/3<663::AID-NME787>3 $.0 . \mathrm{CO} ; 2-8$

[16] Sladeka, J., Sladeka, V. and Zhangb, Ch. (2005) Stress Analysis in Anisotropic Functionally Graded Materials by the MLPG Method. Engineering Analysis with Boundary Elements, 29, 597-609.

https://doi.org/10.1016/j.enganabound.2005.01.011

[17] Horgan, C.O. and Chan, A.M. (1999) The Pressurized Hollow Cylinder or Disk Problem for Functionally Graded Isotropic Linearly Elastic Materials. Journal of Elasticity, 55, 43-59. https://doi.org/10.1023/A:1007625401963

[18] Timoshenko, S.P. and Goodier, J.N. (1970) Theory of Elasticity. 3rd Edition, McGraw Hill Book Company, California. 